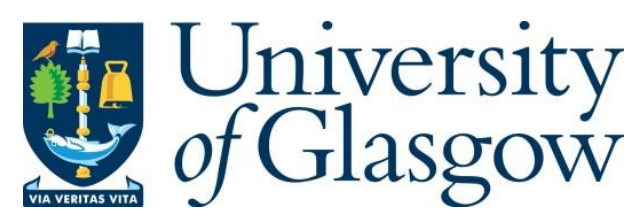

Massey, J. R. et al. (2020) Asymmetric magnetic relaxation behavior of domains and domain walls observed through the FeRh first-order metamagnetic phase transition. Physical Review B, 102(14), 144304.

There may be differences between this version and the published version. You are advised to consult the publisher's version if you wish to cite from it.

http://eprints.gla.ac.uk/223723/

Deposited on: 11 November 2020

Enlighten - Research publications by members of the University of Glasgow http://eprints.gla.ac.uk 


\title{
Asymmetric Magnetic Relaxation behavior of Domains and Domain Walls Observed Through the FeRh First-Order Metamagnetic Phase Transition
}

\author{
Jamie R. Massey, ${ }^{1, *}$ Rowan C. Temple, ${ }^{1}$ Trevor P. Almeida, ${ }^{2}$ Ray Lamb, ${ }^{2}$ Nicolas A. Peters,,${ }^{1,}$ Richard P. Campion, ${ }^{4}$ \\ Raymond Fan, ${ }^{5}$ Damien McGrouther, ${ }^{2}$ Stephen McVitie, ${ }^{2}$ Paul Steadman, ${ }^{5}$ and Christopher H. Marrows ${ }^{1, \dagger}$ \\ ${ }^{1}$ School of Physics and Astronomy, University of Leeds, Leeds LS2 9JT, United Kingdom. \\ ${ }^{2}$ SUPA, School of Physics and Astronomy, University of Glasgow, Glasgow, G12 8QQ, United Kingdom. \\ ${ }^{3}$ School of Electronic and Electrical Engineering, \\ University of Leeds, Leeds, LS2 9JT, United Kingdom. \\ ${ }^{4}$ School of Physics and Astronomy, University of Nottingham, Nottingham, NGr 2RD, United Kingdom. \\ ${ }^{5}$ Diamond Light Source, Chilton, Didcot, OX11 ODE, United Kingdom.
}

(Dated: October 1, 2020)

\begin{abstract}
The phase coexistence present through a first-order phase transition means there will be finite regions between the two phases where the structure of the system will vary from one phase to the other, known as a phase boundary wall. This region is said to play an important but unknown role in the dynamics of the first-order phase transitions. Here, by using both x-ray photon correlation spectroscopy and magnetometry techniques to measure the temporal isothermal development at various points through the thermally activated first-order metamagnetic phase transition present in the near-equiatomic FeRh alloy, we are able to isolate the dynamic behavior of the domain walls in this system. These investigations reveal that relaxation behavior of the domain walls changes when phase coexistence is introduced into the system and that the domain wall dynamics is different to the macroscale behavior. We attribute this to the effect of the exchange coupling between regions of either magnetic phase changing the dynamic properties of domain walls relative to bulk regions of either phase. We also believe this behavior comes from the influence of the phase boundary wall on other magnetic objects in the system.
\end{abstract}

\section{INTRODUCTION}

The dynamic behavior of second-order phase transitions is well understood, owing largely to the critical scaling of the correlation length of thermal fluctuations approaching the temperature associated with the phase transition $^{1-3}$. However, due to the presence of latent heat the same behavior is not expected through firstorder phase transitions ${ }^{1}$. As such, the dynamics of firstorder phase transitions are not as well understood and so remain a topic of active investigation ${ }^{4-8}$. Recent breakthroughs in imaging techniques, capable of tracking various material properties, has led to a surge of interest in materials that exhibit first-order phase transitions ${ }^{4,6,7}$. These investigations focus on the quasi-static development of the first-order phase transition dynamics. They show that first-order phase transition systems demonstrate critical scaling behavior of both the domain size $\mathrm{e}^{4,7}$, and the phase boundary wall ${ }^{4,6}$, which acts to blur the once definitive line between the two phase transition classifications. The coupling between regions of the two phases is cited as the source of this quasi-second-order behavior $^{4-8}$.

Another interesting aspect of phase transition dynamics is their temporal relaxation behavior ${ }^{3,8,9}$. Recently, quasi-second-order behavior has been observed in the phase-ordering and relaxation times in a Mott insulatormetal transition system ${ }^{8}$. Despite this wave of recent interest in first-order phase transition dynamics, the role of the phase boundary wall in these proceedings remains unclear. Very little is known about this region, aside from the critical scaling of the size of the phase boundary wall approaching the transition temperature ${ }^{4,6}$. It is said to play a key, but as yet unknown, role in the evolution of the first-order phase transitions ${ }^{10,11}$.

X-Ray Photon Correlation Spectroscopy (XPCS) has been used to study the relaxation dynamics of domain walls in magnetic systems ${ }^{3,9,12-15}$. The FeRh alloy is a material that, in a specific composition range ${ }^{16}$, undergoes a coupled first-order magnetostructural phase transition from an antiferromagnetic (AF) to a ferromagnetic (FM) state when heated through a transition temperature, $T_{\mathrm{T}}$, that is $\sim 380 \mathrm{~K}$ in bulk ${ }^{17-20}$. The coupled magnetic, charge and structural transitions ${ }^{21-24}$ in this material make it an ideal candidate for use in a plethora of possible magnetic data storage architectures ${ }^{11,25-30}$. Here, by comparing measurements of the isothermal relaxation behavior through the phase transition performed using XPCS and magnetometry techniques we are able to isolate the relaxation behavior of the domain walls. These investigations reveal that the dynamic behavior of domain walls where phase coexistence is present is different to both the FM/AF domain walls and the nucleation and growth of magnetic domains. We believe this behavior emanates from the influence of the phase boundary wall on other objects in the system and that the change in behavior compared to these other objects is due to the influence of interphase exchange coupling that accompanies the phase coexistence in this system. 


\section{EXPERIMENTAL DETAILS}

\section{A. Sample Growth and Characterization}

The sample used in this experiment was a $100 \mathrm{~nm}$ thick FeRh layer grown on a $100 \mathrm{~nm}$ thick NiAl buffer layer deposited using DC magnetron sputtering on a molecular beam epitaxy-grown $\operatorname{GaAs}(25 \mathrm{~nm}) / \operatorname{AlAs}(25 \mathrm{~nm}) / \mathrm{GaAs}$ heterostructure substrate. $\mathrm{NiAl}$ is also a B2-ordered material and the layer used here improves the stability of the FeRh layer and promotes epitaxial growth ${ }^{31}$. The substrate was annealed overnight at $400^{\circ} \mathrm{C}$, after which temperature the NiAl layer was deposited. The system was then heated to $600^{\circ} \mathrm{C}$ where the FeRh layer was grown. The sample was then annealed in situ for 1 hour at $700^{\circ} \mathrm{C}$. No capping layer is used throughout this work as FeRh is robust against oxidation due to the high $\mathrm{Rh}$ content. Structural characterization of the as-grown film was performed using ambient temperature x-ray diffraction (XRD) and is shown in Fig. 1(a). The observation of the (001) and (002) reflections for both the $\mathrm{NiAl}$ and FeRh layers demonstrates chemical order in both layers. Further analysis yields values of the room temperature lattice constant, which have been averaged across both of the peaks present in Fig. 1(a), to be $a=2.981 \pm 0.004$ $\AA$ and $a=2.869 \pm 0.001 \AA$ for FeRh and NiAl respectively.

The expected magnetic transition of the as-grown sample is evident when measured using a SQUID-vibrating sample magnetometer (SQUID-VSM) in a $1 \mathrm{~T}$ in-plane applied field, shown by the black line in Fig. 1(b). The transition temperature of FeRh is sensitive to the application of external field ${ }^{10}$. Therefore, to be able to directly compare the magnetometry with the XPCS measurements (shown in the Results section) which are performed without an external field applied the temperature axis in this figure has been corrected to account for this sensitivity of the transition to externally applied magnetic field. This is case throughout the remainder of this work. The calculation used for this correction is included in the supplementary information ${ }^{10,32}$.

As the magnetic domains in FeRh are of $\mu \mathrm{m}$ dimensions ${ }^{33,34}$, the $Q$ range in which they are found falls within the regime only accessible through transmission experiments. As such, the as-grown film was made into a membrane suitable for $\mathrm{x}$-ray transmission measurements using a HF etching process. This was performed following the method outlined in Ref. 35. The substrate was chosen for its known etching chemistry. By performing the etch in this manner, it is possible to destroy the AlAs layer without harming the rest of the sample. This creates a free standing $\mathrm{FeRh}(100 \mathrm{~nm}) / \mathrm{NiAl}(100 \mathrm{~nm}) / \operatorname{GaAs}(25$ $\mathrm{nm}$ ) layer, which was subsequently captured between two $\mathrm{Cu}$ TEM grids to provide an x-ray transparent sample of B2-ordered FeRh.

After undergoing the etching process to be made into a soft x-ray transparent membrane, the phase transition of the membrane sample was measured in a $1 \mathrm{~T}$ magnetic
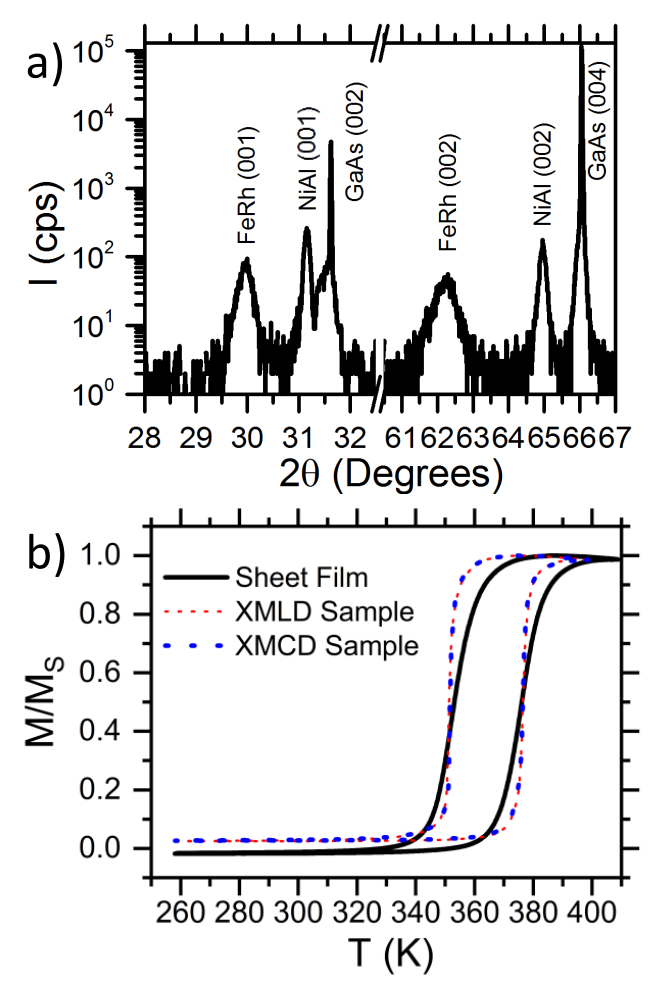

FIG. 1. FeRh thin film sample characterization. (a) Ambient temperature XRD scan with indexed Bragg peaks. The presence of both the (001) and (002) reflections for both $\mathrm{NiAl}$ and FeRh indicates the presence of chemical order in both layers. (b) Magnetometry traces plotted against the corrected temperature $T$ through the range of the transition taken with a $1 \mathrm{~T}$ field applied in the film plane. The black line shows the behavior of the as-grown sample whilst still attached to the substrate, whilst the red and blue lines show the sample behavior after being made into a membrane. These two lines show samples that were used in the XMLD and XMCD experiments, respectively, which are close to indistinguishable.

field applied within the film plane and is shown by the coloured lines in Fig. 1(b). There are two membrane samples here, one used for the experiments concerned with each type of x-ray magnetic dichroism, namely: X-ray Magnetic Circular Dichroism (XMCD) and X-ray Magnetic Linear Dichroism (XMLD). These techniques will be explained in more detail in the results section. The two samples come from the same parent film, and it is clear from Fig. 1(b) that the magnetic transition is still present in the membrane samples after the etching process and that it has become considerably sharper. As the magnetic transition of the as-grown and membrane samples were measured using the same conditions, the difference in the behavior of the two samples comes as a result of removing the substrate, and therefore the strain on the film from the lattice mismatch with the substrate. The two membrane samples have indistinguishable meta- 
magnetic transitions.

\section{B. Soft X-Ray Methods}

The XPCS measurements were carried out at the I10 beamline of the Diamond Light Source. A $20 \mu \mathrm{m}$ radius pinhole is placed in the beampath in front of the samples to provide the coherent light required to generate the speckle pattern ${ }^{3,12}$. To increase the scattering from the magnetic parts of the sample, these measurements were performed with photon energies at the Fe $L_{3}$ resonance. This is measured to be between 706.4-707.2 eV at $400 \mathrm{~K}$ depending on the type of dichroism used. The measurements shown in this work were collected over 3 beamtimes, two of which focus on the XMCD measurements and the third which focusses on XMLD. The beam energy used in the three beamtimes were 707, 707.4 and $706.2 \mathrm{eV}$, respectively. The characterization of the $\mathrm{Fe}$ $\mathrm{L}_{3}$ edge for this sample, and the position of the energy used in each beamtime relative to this, can be seen in the supplementary information.

The images were taken using a 2D charge coupled device (CCD) camera. For each XPCS image consecutive images are performed with opposing helicities (XMCD) or polarization orientations (XMLD) and are combined in post-processing to increase the signal, as per the method outlined by Fischer et al. ${ }^{36}$. In this protocol, the intensity of each image of the final image, $I$, is calculated using

$$
I=I^{+}-I^{-}
$$

where $I^{+}$is the intensity of the image taken with a given helicity or linear polarization and $I^{-}$is the image taken using the opposite helicity or linear polarization. An example of one of these images, taken after cooling to $390 \mathrm{~K}$ using circularly polarized light, can be seen in Fig. 2(a).

Each image series consists of 100 images calculated in this way and are taken over 90-120 minute periods, depending on the type of dichroism used. The sample was thermally cycled between each measurement to reset the domain structure. For measurements performed on the cooling arm the system was cycled into the fully FM phase $(400 \mathrm{~K})$ and then cooled to the desired temperature. For measurements on the heating branch the system was cycled into the fully AF phase $(270 \mathrm{~K})$ and then heated to the desired measurement temperature. All XPCS measurements took place in the absence of a magnetic field. Also included in the data are measurements performed using only a single helicity of circularly polarized light. This occurred after an issue with the undulator during the first beamtime. The signal is much weaker for these measurements than it is for the measurements where the XMCD protocol can be used to boost the signal. These measurements use between 4090 images taken approximately 1 minute apart and are included in this analysis as the values extracted from fitting the data are comparable to the measurements with larger signals.

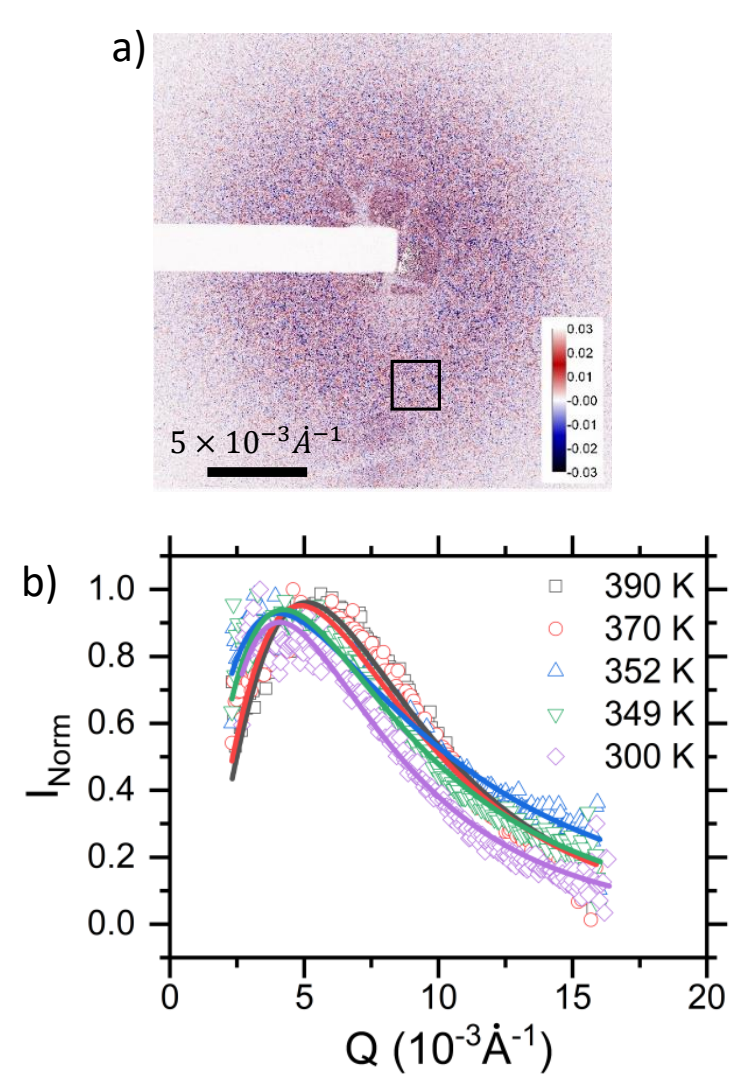

FIG. 2. Resonant magnetic X-Ray scattering. (a) Image taken on the $\mathrm{Fe}_{3}$ resonance edge using circularly polarized light after cooling to $390 \mathrm{~K}$, showing a clear small angle scattering ring with speckle. The shadow cast by the beamstop and some direct transmission though the pinhole and crossed TEM grids is visible in the centre of the image. The colour here represents the intensity of the difference image, with blue regions having a higher intensity of one polarization, whilst the red regions have a higher intensity from the other. (b) Examples of the radial intensity profiles (points) and the fit of a log-normal distribution to the data (lines) taken at various temperatures on the cooling branch using linearly polarized light.

\section{RESULTS}

\section{A. Structural Analysis}

To track the behavior of the magnetic structure within the scattering ring the first image of each series was taken and its radial average was calculated. This procedure was performed after any potential artefacts within the image, such as: the grid, the back of the camera and any holes in the sample holder, are removed. $Q=0$ is taken to be the centre of mass of the image. The XPCS measurements of the dynamic behavior were performed on a $200 \times 200$ pixel box centered around the peak in structural analysis, an example of which can be seen in Fig. 2(a).

Examples of these radial average of the intensity pro- 
files $I(Q)$ for the first image of each of the XPCS sets, where $Q$ is the wavevector transfer, can be seen for various temperatures when cooling performed using linearly polarised light in Fig. 2(b). This reveals a peak which corresponds to correlations in the structure factor ${ }^{37,38}$. These are presented in normalised form as $I_{\text {Norm }}(Q)=$ $\left(I(Q)-I_{\text {Min }}\right) /\left(I_{\text {Max }}-I_{\text {Min }}\right)$. A log-normal distribution was fitted to each data set to identify the position of the peak $^{36}, Q_{\text {Peak }}=Q_{0} e^{-\omega^{2}}$, where $Q_{0}$ is the centre of the distribution and $\omega$ is the logarithm of the full width at half maximum (FHWM) of the peak. It is then possible to extract the spatial lengthscale associated with the peak, $d=2 \pi / Q_{\text {Peak }}$. The results of these fittings for all measurements taken using both circular and linear polarisation are shown in Fig. 3. By using both XMCD, which is sensitive to the behavior of the FM domains only, and XMLD, which is sensitive to the orientation of the spinaxis of the material and can access both FM and AF order $^{39}$, we are able to access the behavior of both the $\mathrm{AF}$ and FM phases, giving a more holistic understanding of the system.

Fig. 3(a) shows measurements taken using circular light for both heating and cooling branches. These reveal an increase towards a peak value at $d \sim 400 \mathrm{~nm}$ when heating which occurs at around $377 \mathrm{~K}$. The dashed lines here mark the position of the transition midpoint, $T_{\mathrm{M}}$, calculated as the steepest point in the magnetometry trace in Fig. 1(b). This convention is used throughout this work and should be taken to be the case unless specified otherwise. At temperatures in excess of $T_{\mathrm{M}} d$ is seen to decrease again in Fig. 3(a), falling to $150 \mathrm{~nm}$ at $400 \mathrm{~K}$. A similar behavior is seen when cooling, though $d$ is seen to be constant at around $150 \mathrm{~nm}$ until the temperature falls below $350 \mathrm{~K}$ after which it is seen to increase, also to about $300 \mathrm{~nm}$, before just starting to drop. These measurements performed during cooling also see the peak value occurring for $T=T_{\mathrm{M}}$. In order to understand these findings it is necessary to consider the development of the magnetic domain structure through the transition.

Previous real-space imaging has shown that FM domains in FeRh nucleate as flux closed structures around $200 \mathrm{~nm}$ in diameter on heating out of the fully AF phase $^{33,34}$. As the transition progresses these domains begin to agglomerate, with the final FM domain state being $\mu \mathrm{m}$ dimension stripes in the absence of externally applied magnetic field, in the fully FM regime ${ }^{33,34}$. The $Q$ range available in this experiment yields sensitivity to objects between 100- $400 \mathrm{~nm}$ in size and so scattering from these $\mu \mathrm{m}$ stripes in not accessible in this experiment. However, scattering across the domain wall between the domains may be accessible. In this system, there are two types of domain wall scattering to consider: i) that between adjacent FM domains and ii) those between domains that are separated by regions of $\mathrm{AF}$ material. The lengthscale associated with scattering from these two objects would have different temperature dependencies through the transition. Given the limited $Q$ range and the changing nature of the domain structure in this experiment, it may be expected that the nature of the scatterer changes across the transition.

To help aid the discussion, diagrams of the proposed structure of the magnetic states through the transition are included in panels (b)-(d) in Fig. 3. The blue regions depict regions of AF material whilst the red and yellow regions are FM domains with their magnetization aligned in opposite directions. The black bars are used to indicate the source of the scattering object at each stage. It is worth noting here that as it was not possible to reconstruct the domain patterns from the scattering patterns, all the discussion surrounding the domain evolution is interpreted from previous real-space imaging experiments $^{33,34,40-42}$.

Considering the measurements performed using circular light in Fig. 3(a), when $T<T_{\mathrm{M}}$ the measured size of the scattering object is consistent with the size of the FM domains seen in these previous works ${ }^{33,34,40}$. It is therefore reasonable to think that the scattering objects are the FM domains that have nucleated from the AF phase as the transition begins, which is shown by Fig. 3(b). On heating it is known that the size of the domains would increase which is reasonable up to $T_{\mathrm{M}}$, but is contradictory to the behavior seen here at higher temperatures. Nevertheless, it is also known that there will still be regions of AF material present between these FM domains $33,34,40-42$ which will shrink when approaching the fully FM phase, therefore decreasing the distance between FM domains. These gaps between the FM phase regions now become the scattering objects, as shown in Fig. 3(c). When cooling from high temperatures $d$ is invariant down to about $360 \mathrm{~K}$. These temperatures are consistent with the fully FM phase when measured using magnetometry and so the scattering in this regime is believed to correspond to domain walls between FM regions with different magnetization directions, as seen in Fig. 3(d). Approaching the transition the rise and fall in $d$ as it passes through a peak at $T_{\mathrm{M}}$ corresponds to the processes on the heating branch in reverse.

Turning to the measurements using linear light in Fig. 3(e), the overall behavior is consistent with peaks in $d$ appearing at $T_{\mathrm{M}}$ on each branch although it is less pronounced on the cooling branch. The length scales extracted for $T>T_{\mathrm{M}}$ appear to be consistent with the corresponding points measured using circular light. Therefore, the nature of the scatterers in this region is believed to be the same in both measurements. However, the behavior of the two diverges for $T<T_{\mathrm{M}}$ where the measurements taken using linearly polarized light appear to be constant at $d \approx 150 \mathrm{~nm}$ down to temperatures of $300 \mathrm{~K}$ for both transition branches. This behavior is consistent with that seen in the only other previous magnetic imaging experiments on the $\mathrm{AF}$ phase of $\mathrm{FeRh}^{43}$ and hence this change in $d$ is attributed to a change in scatterer at $T_{\mathrm{M}}$. At $300 \mathrm{~K}$ the system would exhibit only a $4 \%$ FM volume fraction. Therefore, as XMLD is sensitive to the presence of $\mathrm{AF}$ materials, the change in scatterer at this stage is believed to be AF domain structures. This 


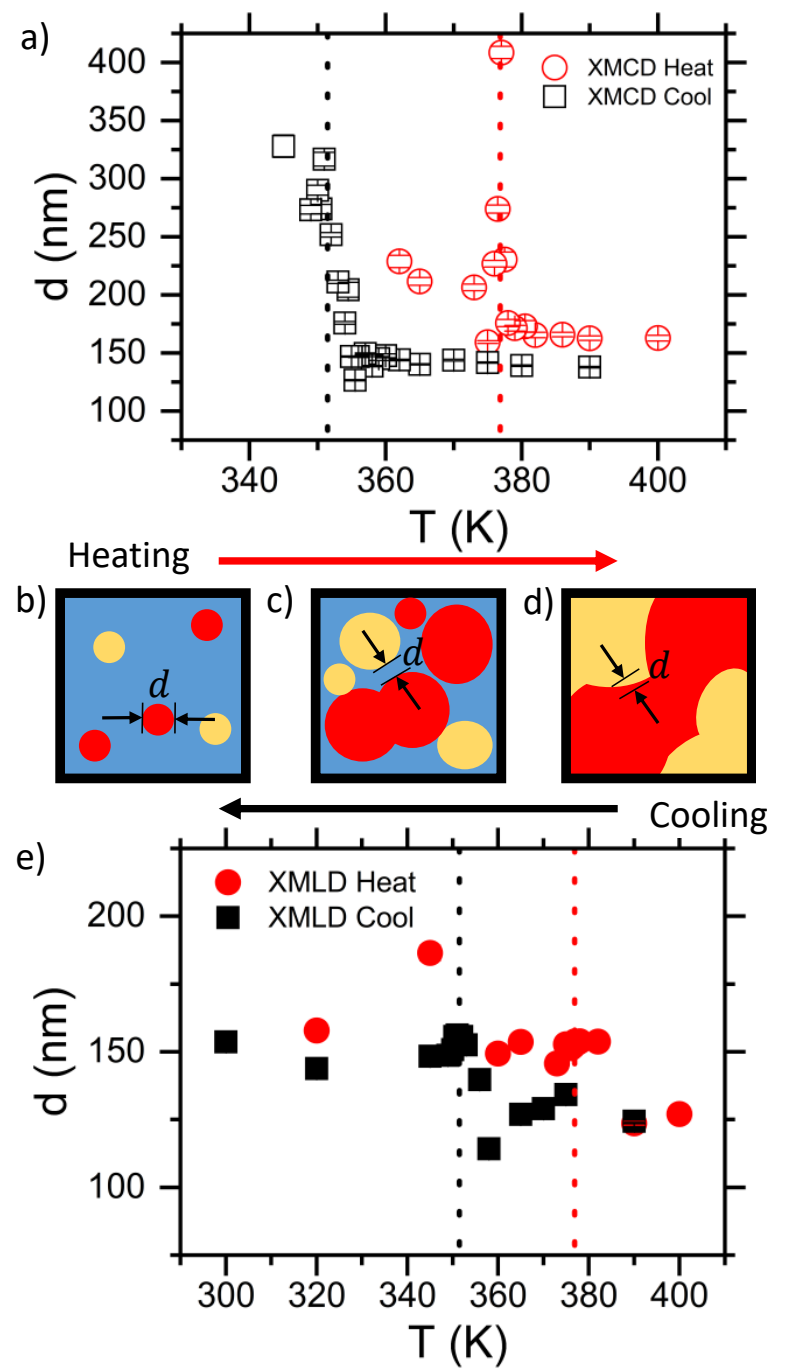

FIG. 3. Temperature dependence of the peak in the radial distribution. (a) and (e) show the length $d$ that corresponds to the position of the peaks seen in the radial intensity profiles, $Q_{\text {Peak }}$, against $T$ for measurements performed using circularly (labelled XMCD) and linearly (labelled XMLD) polarized light respectively. The dashed lines in these panels show the position of the transition midpoint, $T_{\mathrm{M}}$, for each branch, extracted from the magnetometry. Note the different $T$ scales on the abscissae of the two graphs. Panels (b) - (d) show cartoons of the magnetic state at various points through the transition. In these diagrams, the blue depicts AF material and the red and yellow regions are FM domains with the magnetization oriented in opposite directions. The arrows are used to indicate the temperature sweep direction.

behaviour mirrors the scattering from FM domain structures at the highest temperatures for the circular light measurements.

\section{B. X-Ray Photon Correlation Spectroscopy}

XPCS follows the temporal correlations of fine structure present in diffraction features, known as speckle ${ }^{12}$. Panels (a)-(e) of Fig. 4 shows example speckle patterns taken through the measurement time within the black box shown in Fig. 2(a). The changing speckle pattern is indicative of a varying magnetic state through the measurement time. Variations in the speckle intensity are due to fluctuations in the domain walls in magnetic systems ${ }^{3,9,15}$.

To quantify the extent of these changes the temporal auto-correlations for each image series performed at a given temperature are calculated using a $g_{2}$ function. This function is calculated for a series of images separated by a time delay $\tau$ and takes the form ${ }^{3,9,12,13,15}$

$$
g_{2}(\tau)=\left\langle\frac{\langle I(\mathbf{Q}, t) I(\mathbf{Q}, t+\tau)\rangle_{t}}{\langle I(\mathbf{Q}, t)\rangle_{t}^{2}}\right\rangle_{\mathbf{Q}}
$$

where $I(\mathbf{Q}, t)$ is the intensity at position $\mathbf{Q}$ at time $t$ and where $\langle\ldots\rangle_{t, \mathbf{Q}}$ denotes an average over $t$ or $\mathbf{Q}$. Here, the $g_{2}$ function is calculated for each individual pixel, which corresponds to a particular value of $\mathbf{Q}$. These are then averaged over the entire image to give the final $g_{2}$ function. $\tau$ is taken to be integer multiples of the time between images. Generally, it is possible for the $g_{2}$ functions to have an explicit $Q$ dependence ${ }^{44}$, though this is not the case for this experiment - see the supplementary information for more details. This $Q$ dependence is not considered for the remainder of this work. Example $g_{2}$ functions calculated for various points on the cooling branch measured using XMCD can be seen in Fig. 4(f).

To extract the dynamic behavior, the $g_{2}$ function is fitted by a stretched exponential model written as

$$
g_{2}(\tau)=1+A \cos (\omega \tau) e^{-\left(\frac{\tau}{\lambda}\right)^{\beta}},
$$

where $A$ is the speckle intensity or correlation amplitude, $\beta$ is the stretching exponent and $\lambda$ is the relaxation time. Examples of fitting this equation to the $g_{2}$ behaviors can be seen by the solid lines in Fig. 4(f). The development of both $A$ and $2 \pi / \omega$ with temperature can be seen in the supplementary information. As the dynamic behavior of the system is captured by the $\beta$ and $\lambda$ parameters, the extracted values of which for all temperatures for both XMCD and XMLD measurements can be seen in Fig. 5 and Fig. 6 respectively, that is where this rest of the discussion shall focus. Measurements at some temperatures were repeated and what is shown throughout Fig. 5 and Fig. 6 is an error weighted average of all the measurements taken at a given temperature. The fits of equation 3 to the $g_{2}$ functions were performed for times where the value of the calculated $g_{2}$ function is larger than 1 .

This form of the fitting function is known as the heterodyne model and requires the presence of a static reference signal, for which $\omega$ represents the mixing frequency between the dynamic and static signals ${ }^{3,44}$. It is worth 

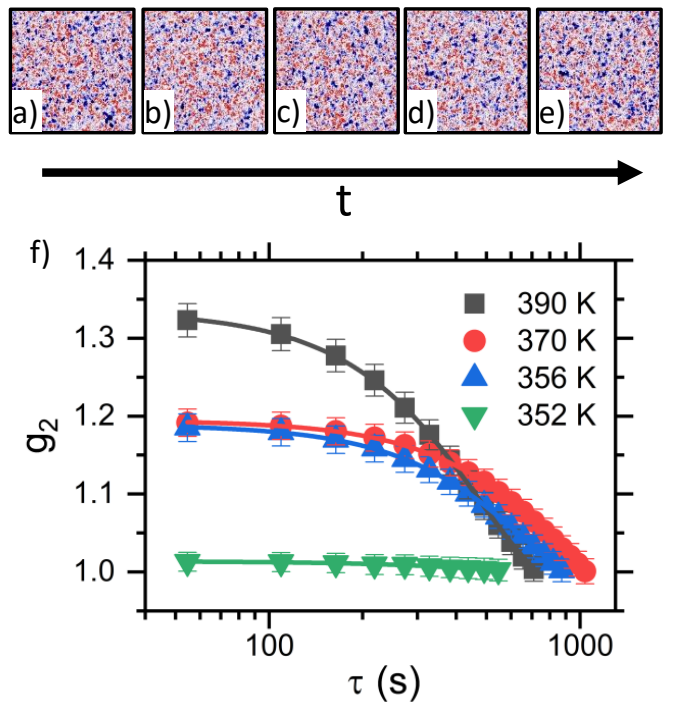

FIG. 4. Temporal correlation analysis. (a) - (e) Example $200 \times 200$ pixel images where the temporal correlation analysis was performed taken at $t=0,1310.4,2620.8,3931.2$ and $5241.6 \mathrm{~s}$ for the measurement performed when cooling to 390 $\mathrm{K}$ using XMCD. The first image of this series is also shown in Fig. 2(a). The speckle pattern clearly changes through the measurement time indicating the presence of dynamic behavior. The black square in panel (a) marks the approximate position where these images were taken. (f) Example $g_{2}$ functions derived from the images (points) and the fits to equation 3 (lines) for various points on the cooling branch of the phase transition taken using circularly polarized light.

mentioning here that this model is chosen as the application of a model which does not assume the presence of a static reference signal, known as the homodyne model ${ }^{44}$, yields values of $\beta$ that are difficult to explain physically. More details of why this is the case will be given in the next section. The static reference signal measured here is believed to originate from the resonant charge scattering in the sample also present at the $\mathrm{Fe}_{3}$ edge.

\section{Stretching Exponent behavior}

For investigations performed using XMCD when cooling it can be seen from Fig. 5 (a) that $\beta \approx 1.5$ for measurements performed where $T>T_{\mathrm{M}}$. For measurements performed where $T \leq T_{\mathrm{M}}$ the extracted values of $\beta$ develop a large scatter, meaning it is difficult to discern any coherent trends in the data. A large scatter in the data is also seen in the extracted values of $\beta$ for measurements performed for $T \leq T_{\mathrm{M}}$ on the heating branch of the transition. Again, for measurements performed where $T>T_{\mathrm{M}}$ when heating exhibit $\beta \sim 1.5$ for all measurements. The large scatter seen in the lower temperatures for each transition branch is likely due to the low

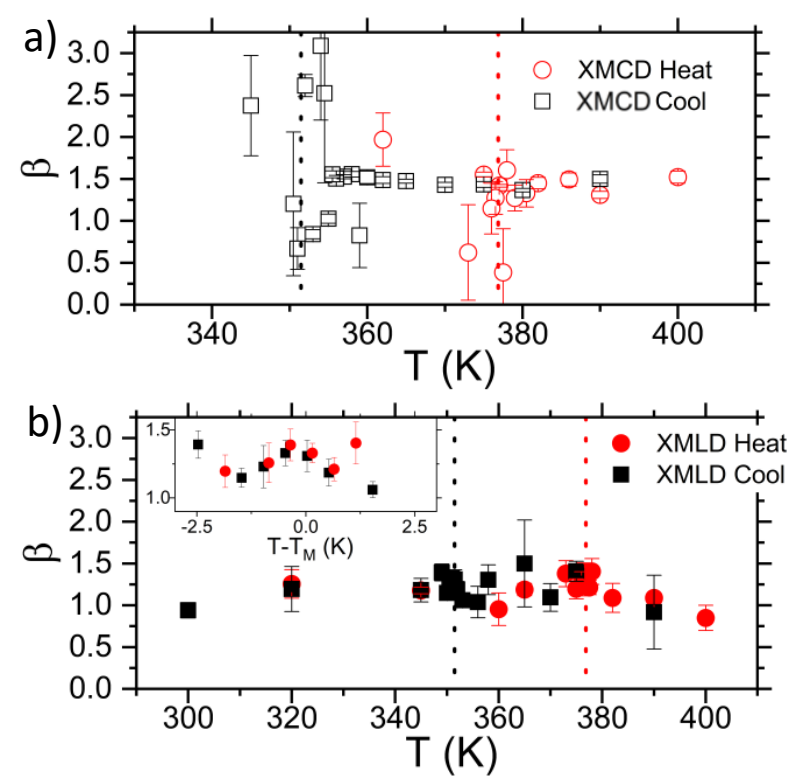

FIG. 5. Stretching exponent behavior. Panels (a) and (b) show the behavior of the stretching exponent, $\beta$, extracted from the fits of equation 3 to the $g_{2}$ functions against temperature, $T$, through the transition for XMCD and XMLD measurements, respectively. The inset in panel (b) shows the behavior of $\beta$ compared to the transition midpoint. The XMCD measurements show $\beta \sim 1.5$ which indicate jammed dynamic behavior, whilst the XMLD measurements appear to show $\beta$ increasing from $\sim 1-1.5$ close to the transition midpoint. The large scatter for measurements performed below $T_{\mathrm{M}}$ when performed using XMCD is attributed to low signal in this region.

volume of FM domains within the system close to the AF phase and the resultant low signal.

The parameter $\beta$ is used to describe the nature of the statistical processes that govern the relaxation of the state $^{9,15,45,46}$. For values of $0<\beta \leq 1$ the relaxation processes are governed by thermal statistical physics ${ }^{45}$, meaning that the dynamic behavior can be described as diffusive $^{9,15}$. Whereas, values of $1<\beta \leq 2$ indicate that the relaxation is governed by Gaussian statistics ${ }^{46}$. In this regime the dynamics are often described as being 'collective' as there are underlying long-range interactions that affect the behavior ${ }^{9,15}$. Systems in which $\beta=1.5$ exhibit what is known as 'jammed' dynamics, meaning that relaxation events are unable to propagate through the system ${ }^{9,15,45-48}$. In magnetic systems the source of this frustration is the inability to fully resolve the Ruderman-Kittel-Kasuya-Yosida (RKKY) exchange coupling present in the system ${ }^{15}$. In the case of FeRh the inability to resolve all the exchange interactions within the region between the two magnetic phases may also contribute to this behavior ${ }^{49}$. It is likely that both factors contribute to the jammed behavior seen in the XMCD measurements in Fig. 5(a). 
The values of $\beta$ extracted from the fits of equation 3 to the $g_{2}$ functions calculated from the XMLD measurements are shown in Fig. 5(b). The development of $\beta$ with temperature is inherently different to that of the XMCD measurements. For temperatures in excess of $T_{\mathrm{M}}$ for both transition branches $\beta \sim 1$ which is indicative of diffusive dynamics ${ }^{9,45}$. When approaching $T_{\mathrm{M}}$ from either direction $\beta$ increases towards $\sim 1.5$ on both transition branches as seen by the inset in Fig. 5(b). Again, for measurements performed at temperatures lower than $T_{\mathrm{M}}$ for both transition branches $\beta$ is mostly consistent with the value 1 within an error bar.

This increase in $\beta$ when approaching $T_{\mathrm{M}}$ for the XMLD measurements coincides with the introduction of FM material into the AF matrix when heating, as well as its removal when cooling. As XMLD is sensitive to both $\mathrm{AF}$ and FM order and the relaxation behaviour around $T_{\mathrm{M}}$ differs from the behaviour of both the AF and FM phases, this change suggests that the properties of the system change fundamentally as phase coexistence is introduced into the system. As this is a system where the AF phase is in direct contact with the FM phase, this variation in the dynamic behaviour with the introduction of phase coexistence suggests that the exchange coupling between the two magnetic phases plays a role in the dynamic behavior of the system. The introduction of exchange coupling between the AF and FM regions would introduce an extra anisotropy energy into the system which would affect the behavior of the spin-axes of both magnetic phases and the magnetic structure in the region between them. This would mean the behavior of the two magnetic phases would become intertwined leading to collective dynamics, as consistent with the experimental data.

\section{Behavior of the Relaxation Time}

As the system jams approaching $T_{\mathrm{M}}$ when measured by XMLD it may be expected that the relaxation time would also increase at this stage. The values of $\lambda$ returned for the fits of Eq. 3 to the $g_{2}$ functions are shown as a function of temperature in Fig. 6(a) and (b) for the XMCD and XMLD experiments, respectively. $\lambda$ is typically several hundred to one thousand seconds without any clear trend through the temperature range here.

Typically, there are three models used to explain the relaxation behaviors of magnetic systems ${ }^{2,3,9,12}$ : The first is the Vogel-Fulcher-Tamann law ${ }^{2,3}$, which describes the behavior of fluctuations as they approach a glass transition temperature. This model is used to describe a thermally activated process with an activation energy that varies with temperature $^{15}$. However, this model is asymmetric around the critical temperature which is not the case for the data here.

The second model is that of the Arrhenius model, which is used to describe systems where a relaxation process is governed by a temperature independent activation energy, $E_{\mathrm{A}}{ }^{15}$. The relationship between the relaxation rate $\lambda^{-1}$, where $\lambda$ is the relaxation time, and the temperature, $T$, is given by:

$$
\ln \left(\lambda^{-1}\right)=\ln \left(\lambda_{0}^{-1}\right)-\frac{E_{\mathrm{A}}}{k_{\mathrm{B}} T},
$$

where $\lambda_{0}^{-1}$ is the relaxation rate at $T=0$ and $k_{\mathrm{B}}$ is the Boltzmann constant ${ }^{50}$. Arrhenius analysis was performed on the XPCS measurements and the results are shown in Fig. 6 for measurements performed using both XMCD (panel (c)) and the XMLD (panel (d)).

The solid lines in Fig. 6(c) and (d) are fits of Eq. 4 to the behavior of the relaxation time extracted from the XPCS measurements. This model is found to describe well the behavior of the measurements performed when heating, yielding $E_{\mathrm{A}} / k_{\mathrm{B}}=4700 \pm 600 \mathrm{~K}$ and $E_{\mathrm{A}} / k_{\mathrm{B}}=1100 \pm 500 \mathrm{~K}$ for the XMCD and XMLD measurements, respectively. The difference here suggests an asymmetry in the nature of the behavior probed, consistent with the difference in the nature of the probe itself. This model yields a negative activation energy for both data sets taken when cooling. This is a non-physical result and implies this model cannot adequately describe the behavior seen in those datasets. Clearly, the inability of the models mentioned thus far to explain the behavior of the cooling branch behaviors requires further investigation.

Another model used to describe relaxation behavior is that of critical slowing down, which describes the relaxation behavior for systems approaching a critical temperature associated with phase transition, $T_{\mathrm{c}}{ }^{2,3}$. The dependence of the relaxation time, $\lambda$, upon its proximity to $T_{\mathrm{c}}$ is given $b^{2,3}$ :

$$
\lambda=\lambda_{0}\left|1-\frac{T}{T_{\mathrm{c}}}\right|^{-z v},
$$

where $z v$ is the critical scaling exponent. The fitting of this equation to the data is shown by the solid lines in Fig. 6(e) and (f) for the XMCD and XMLD investigations, respectively. The fits are performed using $T_{\mathrm{M}}$ extracted from the magnetometry measurements. The extracted values of $z v$ from these fits are shown in Fig. 8. The critical slowing down model is seen to describe the data reasonably well for all datasets. This implies that critical scaling of the relaxation time is observed approaching $T_{\mathrm{M}}$ through the FeRh metamagnetic phase transition when probed using XPCS.

Critical scaling behavior is typically associated with second-order phase transitions where a divergence of the correlation length of thermal fluctuations is seen approaching $T_{\mathrm{c}}{ }^{1,2}$. This divergence brings with it an increase in the activation energy centered around the $T_{\mathrm{c}}$. The same behavior is not expected through first-order phase transitions ${ }^{1}$, though critical scaling of the domain size of a given phase within the other has been seen through various first-order phase transition systems ${ }^{4}$ including $\mathrm{FeRh}^{7}$. However, in this experiment we are un- 

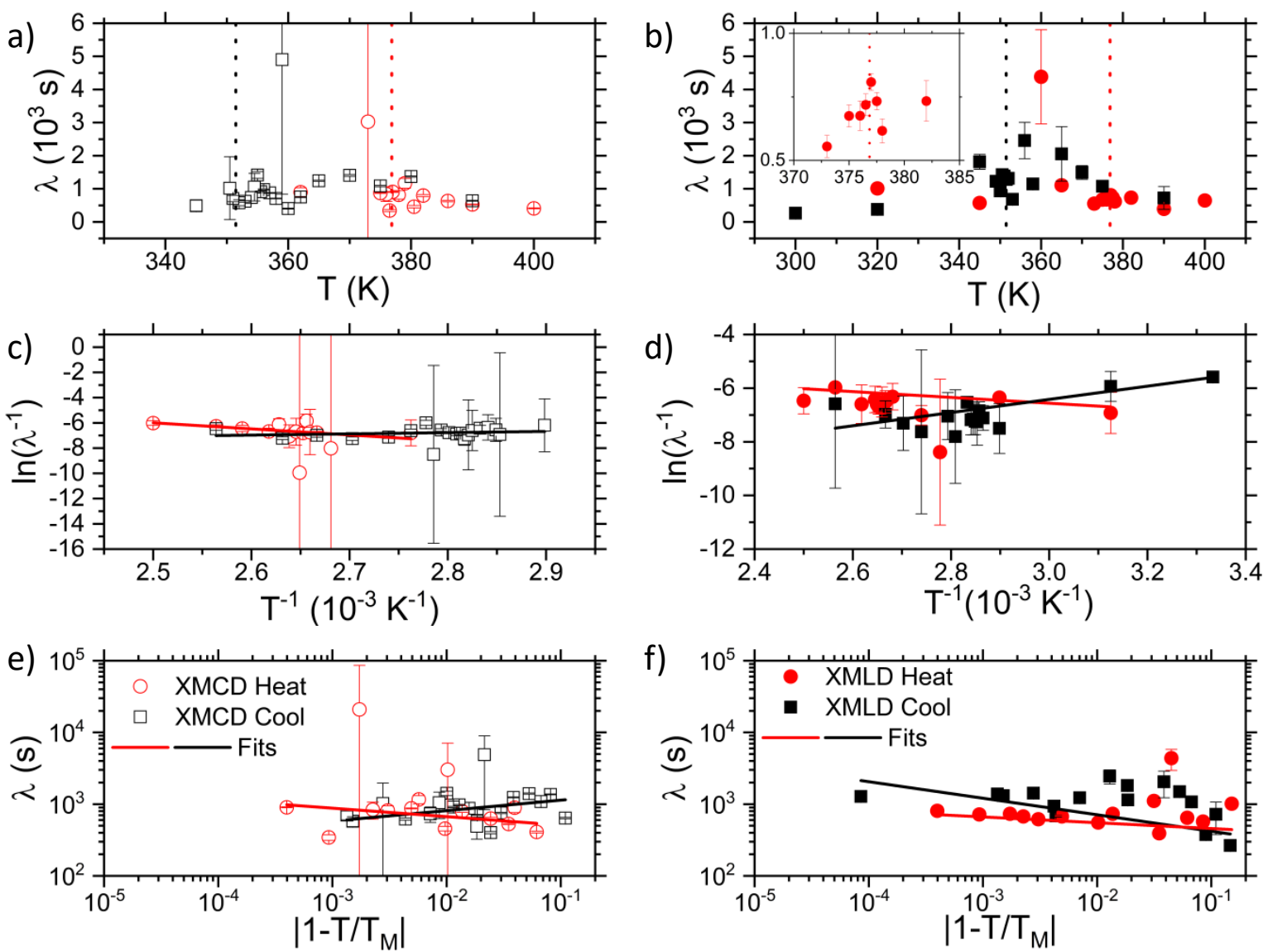

FIG. 6. Behavior of the relaxation time extracted from the XPCS measurements. Panels (a) and (b) show the temperature dependence of the relaxation time, $\lambda$ extracted from measurements performed using XMCD and XMLD measurements, respectively, for measurements performed both when heating and when cooling. There are no clear trend here in the data. Panels (c) and (d) show the data in panels (a) and (b) replotted in line with the Arrhenius equation seen in 4, with the solid lines here being fits of that equation to the data. This model can be used to describe the heating branch behavior well, but leads to a negative activation energy for measurements performed when cooling, which is a non-physical result. Panels (e) and (f) again show data in panels (a) and (b) but this time they are replotted to make it easier to fit the critical slowing down model, seen in equation 5, to the data. Again, the solid lines in these panels are fits of the critical slowing down model to the data. Despite the large scatter in the data, this model appears to describe the behavior of all data sets reasonably well.

able to reconcile the behavior of the relaxation time with the measured length scale (see supplemental material).

The isothermal temporal evolution of the first-order phase transition in FeRh has previously been described using the 'droplet' model ${ }^{50}$, which is used to model systems where one phase forms within the matrix of the other ${ }^{47,50}$. In this scenario there are two main sources of fluctuations: i) the nucleation or annihilation of regions of one phase within the other and ii) fluctuations in the region between the two phases ${ }^{47,50}$. Either pathway could be responsible for the behavior here. So to try and isolate the source of this behavior a similar investigation of the relaxation behavior was performed by measuring the isothermal temporal evolution of the phase transition using a SQUID-VSM, where sensitivity to the domain wall dynamics would be lost to the macroscale behavior of the domain relaxation.

\section{Magnetometry Measurements}

For these measurements, for comparison with the XPCS investigations, the temperature was ramped at 2 $\mathrm{K} \mathrm{min}{ }^{-1}$ and the magnetization was measured for 2 hours immediately after the desired temperature was achieved. To reset the magnetic state between measurements, the sample was thermally cycled using the same protocol as the XPCS measurements. The results of this study are shown in Fig. 7.

The time dependent phase fraction, $\alpha(t)$, is defined as the ratio of the phase changed during the time interval, $t$, and the total available phase fraction available at that temperature and is calculated using ${ }^{50}$ :

$$
\alpha(t)=\frac{M(t)-M_{\mathrm{i}}}{M_{\mathrm{S}}-M_{\mathrm{i}}}
$$

where $M(t)$ is the magnetization at a given time, $t, M_{\mathrm{i}}$ is 

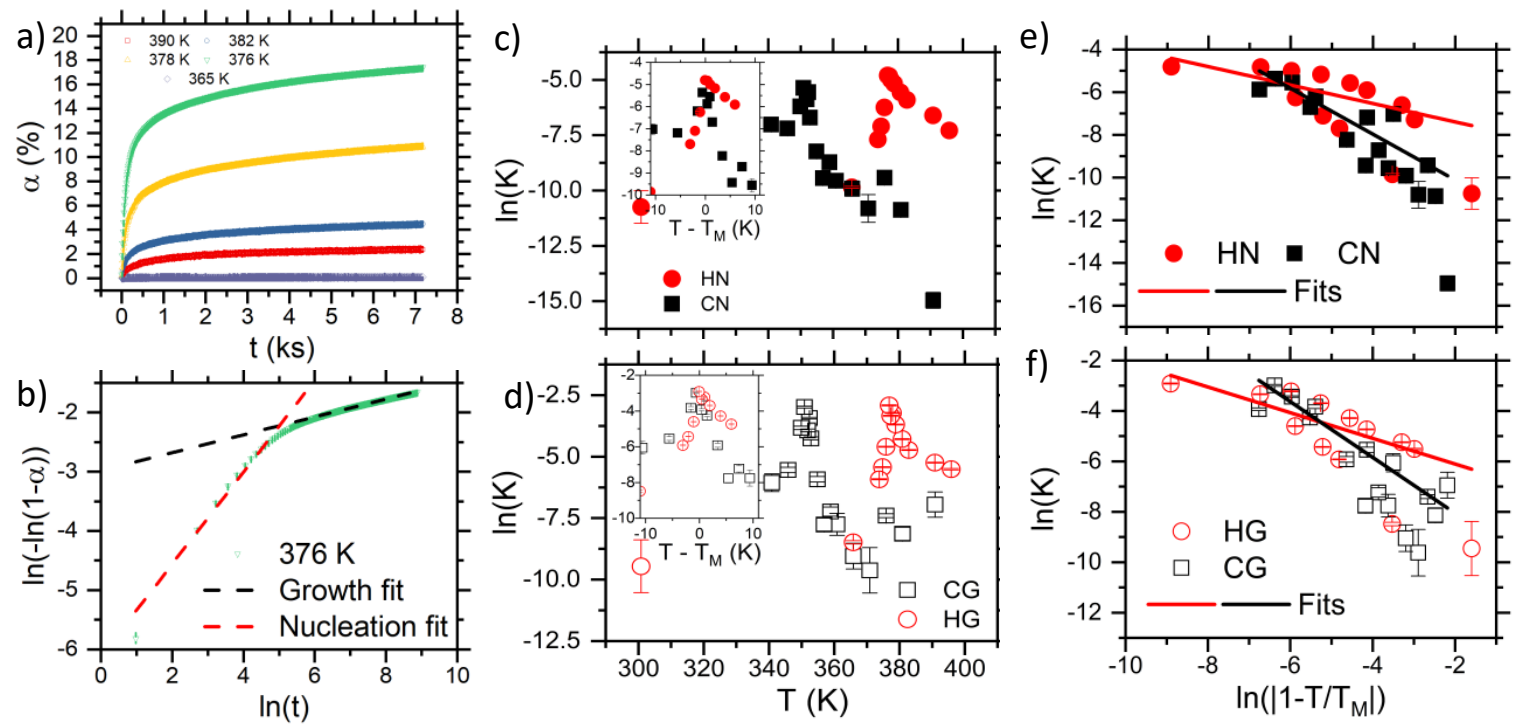

FIG. 7. Magnetic relaxation measured using magnetometry. (a) The evolution of $\alpha$ with time, $t$, for measurements performed at various temperatures when heating. (b) The Avrami analysis (see Methods section for description) for the measurement performed at $376 \mathrm{~K}$. There are two regions where the linear relationship expected is observed here, which are believed to correspond to the nucleation $(\mathrm{N})$ and subsequent growth $(\mathrm{G})$ of domains in the system. The linear fits used to extract information from the two regions are shown by the dashed lines. Panels (c) and (d) show the value of $\ln (K)$ extracted from the Avrami analysis against the corrected temperature, $T$, for the nucleation and growth phases respectively, for measurements performed when heating $(\mathrm{H})$ and cooling $(\mathrm{C})$. The insets in these figures show the behavior of $\ln (K)$ against the deviation in temperature from the transition midpoint, $T-T_{\mathrm{M}}$, zoomed in around $T-T_{\mathrm{M}}=0$. These figures demonstrate an increase in $\ln (K)$ approaching $-T_{\mathrm{M}}=0$, which is indicative of critical speeding up. Panels (e) and (f) show the behavior of $\ln (K)$ extracted for all measurements against the natural logarithm of the reduced temperature $1-T / T_{\mathrm{M}}$, for both the nucleation and growth phases respectively. The solid lines here are fits of the data to the critical slowing down model, the results of which are shown in Fig. 8. Critical speeding up is seen for all measurements sets here.

the magnetization at the beginning of the measurement time, $M_{\mathrm{S}}$ is either the saturation magnetization when heating, or the residual magnetization for measurements performed when cooling.

In the Avrami model the time dependent phase fraction can be written in terms of an exponential probability distribution with a given rate, $K$, after a time $t$ at a given temperature such that ${ }^{50}$ :

$$
\alpha(t)=1-e^{-K t^{n}}
$$

where $n$ is the Avrami exponent and refers to the dimensionality of the changes taking place within the system. It follows then that it is possible to extract both $K$ and $n$ using:

$$
\ln (-\ln (1-\alpha))=\ln (K)+n \ln (t) .
$$

It also follows from this equation that regions of the data where a straight line can be used to accurately describe the behavior means that the system contains a single relaxation process with given dimensionality.

Measurements following the same protocol were also performed on a different $\mathrm{FeRh}$ sample, grown on $\mathrm{MgO}$ with the substrate still attached, through the second- order phase transition approaching the Curie temperature: see the supplementary information for more details.

The temporal evolution of the time dependent phase fraction, $\alpha(t)$, is shown for various temperatures when heating in Fig. 7(a). $\alpha$ clearly varies through the measurement time, with the amplitude of this change peaking for measurements performed near $T_{\mathrm{M}}$. By performing Avrami analysis, which is shown for the $376 \mathrm{~K}$ measurement in Fig. 7(b), it is clear that there are two regions where the expected linear dependence on $\ln (t)$ is observed. This indicates there are two relaxation processes occurring during the measurement time: i) the initial nucleation of FM domains due to thermal equalization of the system (henceforth labelled N) and ii) the subsequent growth $(\mathrm{G})$ of these domains. The value of $\ln (K)$, where $K=\lambda^{-1}$ is the rate constant, extracted from Avrami analysis is shown against $T$ for both the $\mathrm{G}$ and $\mathrm{N}$ regimes in Fig. 7(c) and (d), respectively. All measurements here show that $\ln (K)$ increases for $T \sim T_{\mathrm{M}}$ as seen in the inset where the same data is plotted against $T-T_{\mathrm{M}}$, which is indicative of critical speeding up. This is confirmed by plotting the behavior of $\ln (K)$ in conjunction with the critical slowing down model described by Eq. 5 as seen in panels (e) and (f) of Fig.7. The lines 
here represent a fit to Eq. 5 where the value of $T_{\mathrm{M}}$ is assumed to be that extracted by magnetometry.

Fig. 8 shows a summary of the values of $z v$ extracted for the fits of the critical slowing down model to the measurements performed using the various techniques, including through second-order phase transition at the Curie temperature (see supplemental material). Here, $z v>0$ indicates critical slowing down, whilst critical speeding up is present for $z v<0$. Fig. 8 clearly shows an asymmetry in the nature of the critical scaling for measurements performed using XPCS and magnetometry.

For the measurements performed using XPCS the extracted values of $z v$ are statistically significant from 0 within a $95 \%$ confidence level for both the XMCD heat and XMLD cool datasets. The other two datasets have large scatter compared to their error bars and yield values of $z v$ consistent with 0 within an error bar. It is important to note that the XMCD measurements had low signal for measurements performed for $T<T_{\mathrm{M}}$, which casts doubt on the reliability of this dataset. As it was also found that the Arrhenius model can be used to describe the behavior of the heating branch measurements for both dichroism types, the validity of the $z v$ result extracted for the XMCD measurements when heating is unclear. It is also worth mentioning here that despite the seeming good value extracted of $z v$ from the measurements of the cooling branch using XMLD, it is not clear again whether this model accurately describes the behaviors seen here or that it just fits the fluctuations in the data better than the other models. It is worth noting again here that the measurements performed using XMLD are sensitive to the fluctuations of both magnetic phases, which may make a hinder the ability to describe the behaviour of the system with a single model. Further measurements would be required to say with any certainty whether critical slowing is indeed observed in this system. What is clear from this work however, is that the behavior of the domain wall dynamics is different from that of the domain formation and growth.

\section{DISCUSSION}

Firstly, though there are doubts on the ability of the critical slowing down model to successfully describe the behavior of the XPCS measurements it is clear that the dynamic behavior of the domain wall fluctuations, measured using XPCS, and the nucleation and growth of domains, measured using magnetometry techniques, is different. There is clear evidence of critical speeding up centered around the transition midpoint from the magnetometry measurements whilst the XPCS measurements reveal a much more complicated picture.

$T_{\mathrm{M}}$ is defined as the temperature where phase coexistence is maximised and where the difference in the free energy of the two states is minimised. The rate of domain nucleation would increase for $T \sim T_{\mathrm{M}}$ meaning the critical speeding up seen in the magnetometry measurements can be accounted for easily. It would be expected that the behavior of the domain wall fluctuations would follow the same temperature dependence, where instead the Arrhenius law can be used to describe the behavior seen when heating and none of the models used in this work can describe the behavior of the cooling branch measurements effectively. We can say, however, that the dynamic behavior of the domain walls when measured using XMLD is different in the region where there is phase coexistence to the behavior seen in the nominally AF or FM phase, as evidenced by the change in $\beta$ for $T \sim T_{\mathrm{M}}$. This change in behavior coincides with the introduction of phase coexistence into the system, which implies that the introduction of the exchange coupling into the system has a profound affect on the dynamic behavior of the systems' domain walls.

Previous studies into the nature of the interphase exchange coupling in this material have shown that it develops through the phase transition in a manner consistent with thickness dependent phase transitions in AF/FM bilayer systems ${ }^{32}$. The coupling between phases acts to overcome the exchange energy in the FM regions causing a blurring of the magnetic order in the region between the two phases, known as the phase boundary wall ${ }^{32}$. This structure has been predicted to be an agglomeration of regions of both magnetic phases with dimensions between $1-5 \mathrm{~nm}^{32}$. The size of this object mean it is not possible to observe its influence directly in this experiment, as it falls outside the available $Q$ range. However, we believe that we are seeing the influence of the phase boundary wall on other objects within the system, though further work is required to state this with any certainty. The exact role of this exchange coupling in the dynamic behavior is unclear at this stage, but it may act against the influence of latent heat in the region where the coupling is strongest which would give the phase boundary wall different properties to the bulk regions of either magnetic phase, as seen in this experiment.

There is also evidence that suggests the strength of the interphase exchange coupling in FeRh is dependent on the temperature sweep direction; with the heating branch having a higher interphase exchange coupling than the cooling branch. Therefore, one would expect that the influence of the exchange coupling on the dynamic behaviour of the system would be more pronounced in the heating branch measurements compared to those performed whilst cooling. This may explain why the heating branch measurements are difficult to fit in this experiment, though further work is required to say for certain.

The asymmetries seen in the relaxation behaviour of the heating and cooling branches of the transition is consistent with measurements of the temperature driven domain domains of the system ${ }^{10,43,51-53}$. The physical origin of this imbalance in the nucleation kinetics is unclear. Previous reports have attributed it to the influence of strain in the stabilization of the FM phase ${ }^{10}$ and the robustness of FM order against strain ${ }^{52}$. From this work we 
can state that the exchange coupling plays a role in the dynamic behaviour of the phase boundary wall, which will affect the propagation of domains through the material. The exchange coupling in this system is known to weaken the FM exchange and blur the boundary between magnetic phases, and is stronger when heating than when cooling $^{32}$. This suggests that it would act against the formation and propogation of FM domains when heating and favour the formation of AF domains when cooling. The exchange coupling, therefore, would act to narrow the thermal hysteresis seen in this first-order phase transition. FeRh exhibits a coupled magnetic, structural and electrical phase transition and the thermal hysteresis of the phase transition is likely to be a consequence of competition between all these different aspects of the transition. However, it is clear that the exchange coupling and the subsequent effect it has on the domain kinetics should be considered in microscopic models of first-order phase transition systems going forward.

Little is known about the regions between phases in systems that exhibit first-order phase transitions but it is clear from this work that it plays a role in the dynamic behavior. Given there are many ways that two states in a first-order phase transition system can couple across the boundary including elastic coupling due to $\operatorname{strain}^{8,27}$, magnetic coupling in metamagnetic transitions ${ }^{32,54}$, and charge coupling in metal-insulator systems ${ }^{4,6}$, it may be that this influence of the coupling across the phase boundary can be seen to affect a variety of different firstorder phase transition systems. This work highlights that the influence of interphase coupling should be considered in theories of first-order phase transition dynamics when interested in the smaller scale behavior, as well as demonstrating that the phase boundary wall to be an interesting entity in its own right which requires further study. We hope, as the next generation of synchrotron sources become available it will be possible to resolve the structure of the phase boundary wall in this, and other, first-order phase transition systems.

\section{CONCLUSION}

To conclude, XPCS investigations were performed using both XMCD and XMLD to measure the dynamic behavior of the system through the FeRh metamagnetic phase transition. When probed using XMCD investigations of the static structure of the scattering ring reveal a change in the nature of the scatterer when heating through the transition from domains to domain walls and back again when cooling. Whereas, measurements performed using XMLD show a change in the nature of the scatterer from being the FM material to AF material centered around the transition midpoint.

The dynamic behavior extracted from the XPCS measurements paints a much more complicated picture. Measurements performed using XMCD reveal jammed dynamics where $T>T_{\mathrm{M}}$ and a large scatter below that,

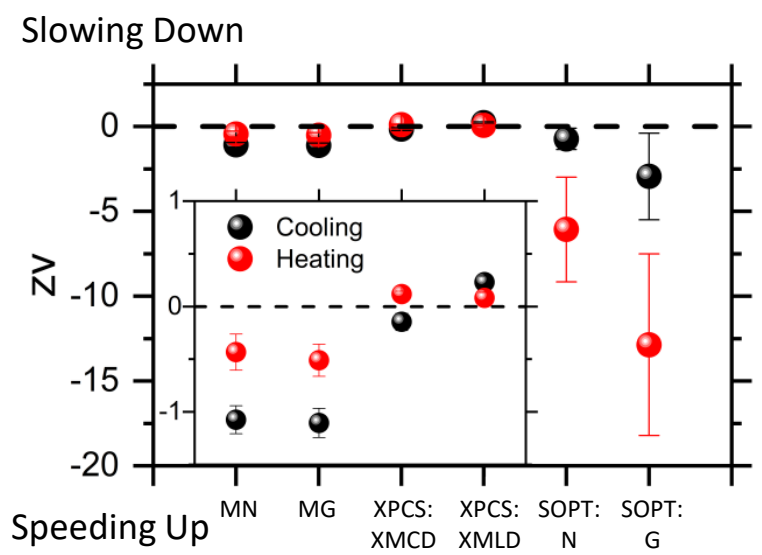

FIG. 8. Summary of critical scaling analysis. The value of the critical scaling exponent, $z v$, is shown for the various measurement techniques here for measurements performed both when heating and whilst cooling. Here, M means measurements performed using magnetometry through the first-order phase transition and SOPT refers to measurements performed using magnetometry through the second-order phase transition approaching the Curie temperature (see supplemental material). The definition of the critical slowing down model here means that $z v>0$ is indicative of critical slowing down, whilst $z v<0$ implies critical speeding up. There is a clear asymmetry between the measurements performed using magnetometry techniques and those performed using XPCS.

most likely due to the low volume of FM material at low temperatures. The nature of the dynamic behavior appears to undergo a transition from diffusive to jammed dynamics centered around $T \sim t_{\mathrm{M}}$. This is attributed to the exchange coupling that accompanies the introduction of phase coexistence into the system as the transition progresses. It is found that the Arrhenius model can be used to describe the behavior of the relaxation time of the measurements performed when heating. Whereas, the behavior of the relaxation time for the cooling branch measurements cannot be described well with any of the Arrhenius, critical slowing down or Vogel-Fulcher-Tammann models.

To try and ascertain the source of these behaviors measurements of the dynamic behavior on the macroscale were performed using magnetometry techniques. These investigations reveal critical speeding up around the transition midpoint, which can be attributed to the reduction in the free energy difference between the two states at this point. The macroscale investigations imply that the behavior measured using XPCS belongs to domain wall fluctuations, where the influence of the interphase coupling on the behavior of the relaxation behavior can be clearly seen in the XMLD measurements. The exchange coupling changes the dynamics of domain walls where there is phase coexistence compared to the domain wall behavior in either the nominally AF or FM phase. We believe that this change in the behavior comes from the phase boundary wall, whose influence we see in the be- 
havior of other objects as it is too small to be measured directly in this experiment. This work highlights the importance of considering the role of the interphase coupling in models of first-order phase transition dynamics on the microscale.

\section{ACKNOWLEDGMENTS}

This work was supported by the Diamond Light Source and the UK EPSRC (grant numbers EP/M018504/1 and $\mathrm{EP} / \mathrm{M} 019020 / 1)$.
* jamie.massey@psi.ch

† c.h.marrows@leeds.ac.uk

1 D. A. Porter and K. E. Easterling, "Phase transformations in metals and alloys," (Van Nostrand Reinhold Publishing, 1981) Chap. 5.

2 C. Djurberg, P. Svedlindh, P. Nordblad, M. F. Hansen, F. Bødker, and S. Mørup, Phys. Rev. Lett. 79, 5154 (1997).

3 S. A. Morley, D. Alba Venero, J. M. Porro, S. T. Riley, A. Stein, P. Steadman, R. L. Stamps, S. Langridge, and C. H. Marrows, Phys. Rev. B 95, 104422 (2017).

4 A. S. McLeod, E. van Heumen, J. G. Ramirez, S. Wang, T. Saerbeck, M. Goldflam, L. Anderegg, P. Kelly, A. Mueller, M. K. Lui, I. K. Schuller, and D. N. Basov, Nat. Physics. 13, 80 (2016).

5 T. Nemoto, R. L. Jack, and V. Lecomte, Phys. Rev. Lett. 118, 115702 (2017).

${ }^{6}$ K. W. Post, A. S. McLeod, M. Hepting, M. Bluschke, Y. Wang, G. Cristiani, G. Logvenov, A. Charnuhka, G. X. Ni, P. Radhakrishnan, M. Minola, A. Pasupathy, A. V. Boris, E. Benckiser, K. A. Dahmen, E. W. Carlson, B. Keimer, and D. N. Basov, Nat. Physics. 14, 1056 (2018).

7 D. J. Keavney, Y. Choi, M. V. Holt, V. Uhlír, D. Arena, E. E. Fullerton, P. J. Ryan, and J.-W. Kim, Sci. Rep. 8, 1778 (2018)

8 S. Kundu, T. Bar, R. K. Nayak, and B. Bansal, Phys. Rev. Lett. 124, 095703 (2020).

9 X. M. Chen, B. Farmer, J. S. Woods, S. Dhuey, W. Hu, C. Mazzoli, S. B. Wilkins, R. V. Chopdekar, A. Scholl, I. K. Robinson, L. E. De Long, S. Roy, and J. T. Hastings, Phys. Rev. Lett. 123, 197202 (2019).

10 S. Maat, J. U. Thiele, and E. E. Fullerton, Phys. Rev. B 72, 214432 (2005).

11 T. Moriyama, N. Matsuzaki, K. J. Kim, I. Suzuki, T. Taniyama, and T. Ono, Appl. Phys. Lett. 107, 122403 (2015).

12 S. K. Sinha, Z. Jiang, and L. B. Lurio, Advanced Materials 26, 7764 (2014).

13 O. G. Shpyrko, E. D. Isaacs, J. M. Logan, Y. Feng, G. Aeppli, R. Jaramillo, H. C. Kim, T. F. Rosenbaum, P. Zschack, M. Sprung, S. Narayanan, and A. R. Sandy, Nature 447, 68 (2007).

14 S. Konings, C. Schüßler-Langeheine, H. Ott, E. Weschke, E. Schierle, H. Zabel, and J. B. Goedkoop, Physical Review Letters 106, 077402 (2011).

15 S.-W. Chen, H. Guo, K. A. Seu, K. Dumesnil, S. Roy, and S. K. Sinha, Phys. Rev. Lett. 110, 217201 (2013).

16 L. Swartzendruber, Journal of Phase Equilibria 5, 456 (1984).

17 M. Fallot and R. Hocart, Rev. Sci. 8, 498 (1939).

18 J. S. Kouvel and C. C. Hartelius, J. Appl. Phys. 33, 1343 (1962).

19 J. S. Kouvel, J. Appl. Phys. 37, 1257 (1966).
20 M. A. de Vries, M. Loving, A. P. Mihai, L. H. Lewis, D. Heiman, and C. H. Marrows, N. J. Phys. 15, 013008 (2013).

21 J. A. Ricodeau and D. Melville, Journal of Physics F: Metal Physics 2, 337 (1972).

22 J.-U. Thiele, M. Buess, and C. H. Back, Appl. Phys. Lett. 85, 2857 (2004).

${ }^{23}$ G. Ju, J. Hohlfeld, B. Bergman, R. J. M. van de Veerdonk, O. N. Mryasov, J.-Y. Kim, X. Wu, D. Weller, and B. Koopmans, Phys. Rev. Lett. 93, 197403 (2004).

24 I. Radu, C. Stamm, N. Pontius, T. Kachel, P. Ramm, J.-U. Thiele, H. Dürr, and C. Back, Phys. Rev. B 81, 104415 (2010).

25 J. U. Thiele, S. Maat, and E. E. Fullerton, Appl. Phys. Lett. 82, 2859 (2003).

${ }^{26}$ R. O. Cherifi, V. Ivanovskaya, L. C. Phillips, A. Zobelli, I. C. Infante, E. Jacquet, V. Garcia, S. Fusil, P. R. Briddon, N. Guiblin, A. Mougin, A. A. Unal, F. Kronast, S. Valencia, B. Dkhil, A. Barthélémy, and M. Bibes, Nat. Mater. 13, 345 (2014).

27 Y. Lee, Z. Q. Liu, J. T. Heron, J. D. Clarkson, J. Hong, C. Ko, M. D. Biegalski, U. Aschauer, S. L. Hsu, M. E. Nowakowski, J. Wu, H. M. Christen, S. Salahuddin, J. B. Bokor, N. A. Spaldin, D. G. Schlom, and R. Ramesh, Nat. Comms. 6, 5959 (2015).

28 X. Marti, I. Fina, C. Frontera, J. Liu, P. Wadley, Q. He, R. J. Paull, J. D. Clarkson, J. Kudrnovský, I. Turek, J. Kuneš, D. Yi, J. H. Chu, C. T. Nelson, L. You, E. Arenholz, S. Salahuddin, J. Fontcuberta, T. Jungwirth, and R. Ramesh, Nat. Mater. 13, 367 (2014).

${ }^{29}$ C. Le Graët, T. R. Charlton, M. McLaren, M. Loving, S. A. Morley, C. J. Kinane, R. M. Brydson, L. H. Lewis, S. Langridge, and C. H. Marrows, APL Materials 3, 041802 (2015).

30 R. C. Temple, M. C. Rosamond, J. R. Massey, T. P. Almeida, E. H. Linfield, D. McGrouther, S. McVitie, T. A. Moore, and C. H. Marrows, arXiv e-prints , arXiv:1905.03573 (2019).

31 D. Kande, S. Pisana, D. Weller, D. E. Laughlin, and J. G. Zhu, IEEE Transactions on Magnetics 47, 3296 (2011).

32 J. R. Massey, K. Matsumoto, M. Strungaru, R. C. Temple, T. Higo, K. Kondou, R. F. L. Evans, G. Burnell, R. W. Chantrell, Y. Otani, and C. H. Marrows, Phys. Rev. Materials 4, 024403 (2020).

33 T. P. Almeida, R. Temple, J. Massey, K. Fallon, D. McGrouther, T. Moore, C. H. Marrows, and S. McVitie, Sci. Rep. 7, 17835 (2017).

34 R. C. Temple, T. P. Almeida, J. R. Massey, K. Fallon, R. Lamb, S. A. Morley, F. Maccherozzi, S. S. Dhesi, D. McGrouther, S. McVitie, T. A. Moore, and C. H. Marrows, Phys. Rev. Materials 2, 104406 (2018).

35 C. Russell, C. D. Wood, A. D. Burnett, L. Li, E. H. Linfield, A. G. Davies, and J. E. Cunningham, Lab on a Chip 13, 4065 (2013). 
${ }^{36}$ P. Fischer, R. Zeller, G. Schütz, G. Georigk, and H. Haubold, Journal de Physique IV 7, 753 (1997).

37 D. S. Sivia, "Elementary scattering theory for x-ray and neutron users," (Oxford University Press, 2011) Chap. 5.

38 K. Bagschik, R. Frömter, J. Bach, B. Beyersdorff, L. Müller, S. Schleitzer, M. H. Berntsen, C. Weier, R. Adam, J. Viefhaus, C. M. Schneider, G. Grübel, and H. P. Oepen, Phys. Rev. B 94, 134413 (2016).

39 J. Stohr and H. C. Siegmann, "Magnetism: From fundamentals to nanoscale dynamics," (Springer Series in Solid State Physics, 2006) Chap. 9.

40 C. Baldasseroni, C. Bordel, A. X. Gray, A. M. Kaiser, F. Kronast, J. Herrero-Albillos, C. M. Schneider, C. S. Fadley, and F. Hellman, Appl. Phys. Lett. 100, 262401 (2012).

41 C. J. Kinane, M. Loving, M. A. de Vries, R. Fan, T. R. Charlton, J. S. Claydon, D. A. Arena, F. Maccherozzi, S. S. Dhesi, D. Heiman, C. H. Marrows, L. H. Lewis, and S. Langridge, N. J. Phys. 16, 113073 (2014).

42 S. O. Mariager, L. Le Guyader, M. Buzzi, G. Ingold, and C. Quitmann, arXiv e-prints , arXiv:1301.4164 (2013).

43 C. Baldasseroni, C. Bordel, C. Antonakos, A. Scholl, K. H. Stone, J. B. Kortright, and F. Hellman, J. Phys. : Cond. Mater. 27, 256001 (2015).
44 W. H. de Jeu, A. Madsen, I. Sikharulidze, and S. Sprunt, Physica B: Cond. Mat. 357, 39 (2005).

45 D. C. Johnston, Phys. Rev. B 74, 184430 (2006).

46 E. W. Hansen, X. Gong, and Q. Chen, Macro. Chem. and Phys. 214, 844 (2013).

47 K. Binder, Reports on Progress in Physics 50, 783 (1987).

48 E. J. Banigan, M. K. Illich, D. J. Stace-Naughton, and D. A. Egolf, Nature Physics 9, 288 (2013).

49 T. A. Ostler, C. Barton, T. Thomson, and G. Hrkac, Phys. Rev. B 95, 064415 (2017).

50 M. Loving, Understanding the Magnetostructural Transformation in FeRh thin films, Ph.D. thesis, The Department of Chemical Engineering, Northeastern University (2013).

51 M. A. de Vries, M. Loving, M. McLaren, R. M. D. Brydson, X. Liu, S. Langridge, L. H. Lewis, and C. H. Marrows, Appl. Phys. Lett. 104, 232407 (2014).

52 V. Uhlíř, J. A. Arregi, and E. E. Fullerton, Nat. Comms. 7, 13113 (2016).

53 J. A. Arregi, M. Horký, K. Fabianová, R. Tolley, E. E. Fullerton, and V. Uhlír, J. Phys. D: Appl. Phys. 51, 105001 (2018).

54 I. Gray, G. M. Stiehl, J. T. Heron, A. B. Mei, D. G. Schlom, R. Ramesh, D. C. Ralph, and G. D. Fuchs, Phys. Rev. Materials 3, 124407 (2019). 